\title{
Microvascular remodeling of nasal mucosa in allergic rhinitis induced by an allergen in Sprague-Dawley rats
}

\author{
J.G. Liu', M.Q. Wang ${ }^{1}$, X.H. Zhu' ${ }^{1}$, Y.H. Liu' ${ }^{1}$ and J.Y. Cai' ${ }^{2}$ \\ ${ }^{1}$ Department of Otorhinolaryngology, \\ The Second Affiliated Hospital of Nanchang University, Nanchang, Jiangxi, China \\ ${ }^{2}$ Department of Anesthesiology, \\ The Second Affiliated Hospital of Nanchang University, Nanchang, Jiangxi, China \\ Corresponding author: Y.H. Liu \\ E-mail: yuehuiliu1@126.com \\ Genet. Mol. Res. 14 (3): 11624-11630 (2015) \\ Received February 5, 2015 \\ Accepted July 24, 2015 \\ Published September 28, 2015 \\ DOI http://dx.doi.org/10.4238/2015.September.28.14
}

\begin{abstract}
This study aimed to observe microvascular changes in the nasal mucosa of Sprague-Dawley (SD) rats with allergic rhinitis (AR) after persistent exposure to an allergen with fluticasone propionate (FP) treatment. Ninety healthy SD rats were randomly distributed into the control group ( $A, N=30)$, the group with continued exposure to an allergen $(B, N$ $=30)$, and FP treatment group $(C, N=30)$. The animals of the persistence group were subjected to persistent exposure to an allergen after 7 weeks of modeling of ovalbumin (OVA) provocation in the nasal mucosa for 16 weeks. At the 8th, 12th, and 16th week after OVA provocation, each group was euthanized at each time point: the FP treatment after OVA provocation, and animals of the control group were not stimulated with OVA and were sacrificed at the same time point. The nasal mucosa of 5 animals from each group was analyzed for the expression of vascular endothelial growth factor (VEGF), and another 5 animals were used to make micro vascular corrosion casts for a scanning electron microscope. The results demonstrate that FP has a strong inhibitory effect on angiogenesis in AR.
\end{abstract}


Inhalation of FP had an antiangiogenic effect through inhibition of VEGF expression but does not completely reverse the remodeling of the nasal mucosa in the short term nor does it have complete control over the expression of VEGF mRNA.

Key words: Allergen; Nasal mucosa; Fluticasone propionate; Microvascular remodeling

\section{INTRODUCTION}

The parenchymatous lesion of allergic rhinitis (AR) is a chronic inflammatory mucosal lesion caused by an allergic reaction; it is characterized by massive infiltration by eosinophils. We previously reported the mucosal lesions and microvascular remodeling features of AR (Liu et al., 2008; Liu and Liu, 2010). The anti-inflammatory therapy based on inhaled corticosteroids (ICS) is widely recommended in the world and plays a decisive role in the airway remodeling process. The most important function of ICS is to inhibit the gene transcription of inflammatory cytokines, but it can also inhibit plasma exudation and mucus secretion during airway inflammation (Barnes, 1995; Lu et al., 2005) so as to promote the airway epithelium remodeling. There were few studies on the role of the remodeling process in the nasal mucosa. In this study, we examined the characteristics of a nasal allergen attack and vascular remodeling after treatment with fluticasone propionate (FP) in AR by means of microvascular corrosion casting and scanning electron microscopy (SEM), respectively, which could provide the theoretical basis for the clinical treatment of AR.

\section{MATERIAL AND METHODS}

\section{Experimental animals}

In total, 90 Specific Pathogen-Free inbred healthy 4- to 5-week-old Sprague-Dawley (SD) rats weighing 130 to $150 \mathrm{~g}$ were obtained from the animal experimental center of Nanchang University. The rats were randomly subdivided into the control group $(A, N=30)$, the group with continued exposure to an allergen $(B, N=30)$, and FP treatment group $(C, N=30)$. The study conforms to the Guide for the Care and Use of Laboratory Animals published by the US National Institutes of Health (NIH Publication No. 85-23, revised in 1996). The study protocol was approved by the Animal Ethics Committee of Nanchang University. The rat model of AR was set up as described previously. The basic sensitization was performed by intraperitoneal injection of $1 \mathrm{~mL}$ allergen suspension containing $0.3 \mathrm{mg}$ ovalbumin (OVA) and $30 \mathrm{mg} \mathrm{Al}(\mathrm{OH})_{3}$ gel in rats: a total of 7 times once every other day. Then $50 \mu \mathrm{L} 5 \%$ OVA suspension drops were administered into bilateral nasal cavities of the rats for excitation: a total of 7 times once a day. Each rat was evaluated after the last stimulation according to the evaluation standards. After the success of the model establishment, $50 \mu \mathrm{L}$ 5\% OVA suspension drops was administered into bilateral nasal cavities of the rats continuously: a total of 8 times, 2 times a week. The experimental rats were subdivided into groups $\mathrm{B}$ and $\mathrm{C}$. The procedure was the same as that mentioned above for group $\mathrm{A}$, with the same volume of saline being used instead of the allergen. In group C, 50- $\mu \mathrm{L}$ FP drops were administered into bilateral nasal cavities after injection of $50 \mu \mathrm{L} 5 \%$ OVA suspension drops into the bilateral nasal cavities half an hour earlier. 


\section{Determination of VEGF expression by RT-PCR}

The nasal septum mucosa was collected in 8,12 , and 16 weeks, with heart bled to death after anesthesia via intraperitoneal injection of $300 \mathrm{mg} / \mathrm{kg} \mathrm{10 \%} \mathrm{chloral} \mathrm{hydrate} \mathrm{in} \mathrm{rats.} \mathrm{Total} \mathrm{RNA}$ from the samples was isolated using an RNA extraction kit following the manufacturer protocol. One microgram total RNA was subjected to reverse transcription using a reverse transcription kit. The primers for RT-PCR were as follows: VEGF forward, 5'-CAGTCGCCTCCAGAGTTTCC-3', reverse: 5'-GTACAAGGAGGCCATCACCAGA-3'; b-actin forward, 5'-AAGTACCCCATTGAACACG -3', reverse: 5'-AGAGGGCAAGAACACAGC-3'. The b-actin gene served as an internal control for normalization of RNA quantity and quality differences among all samples. The procedure for the PCR was as follows: pre-denaturation for $5 \mathrm{~min}$ at $95^{\circ} \mathrm{C}$; denaturation for $30 \mathrm{~s}$ at $95^{\circ} \mathrm{C}$, annealing for $30 \mathrm{~s}$ at $56^{\circ} \mathrm{C}$, extension for $30 \mathrm{~s}$ at $72^{\circ} \mathrm{C}$ for 30 cycles, and extension for $10 \mathrm{~min}$ at $72^{\circ} \mathrm{C}$. The $\mathrm{PCR}$ product was analyzed by means of electrophoresis on a $1 \%$ agarose gel ( $5 \mathrm{~V} / \mathrm{cm} \times 20 \mathrm{~min})$.

\section{Nasal mucosal microvasculature}

SD rats were fixed on the strip board in the supine position after the successful anesthesia with $10 \%$ chloral hydrate; the chest and abdominal cavity were opened, the descending aorta, pulmonary vein and the inferior vena cava were ligated. The heart was cut open from the apex transversely and a catheter was inserted from the left ventricle into the aorta; it was filled with $20 \mathrm{~mL}$ ethyl acetate first until the right atrium showed an outflow of a clear liquid. Then it was filled with the casting agent ( $5 \mathrm{~g}$ self-curing denture, $5 \mathrm{~mL}$ powder, self-curing denture water, $5 \mathrm{~mL}$ dibutyl phthalate, $2 \mathrm{~mL}$ red ink) until the right atrium showed an outflow of a red liquid. The rats were maintained under typical conditions at a ventilated facility for 6-7 days. The head of an SD rat was cut off and placed in $30 \%$ hydrochloric acid: digestion for $\sim 4$ days to soften the tissue. The nasal part was cut out and placed in $50 \%$ hydrochloric acid for 3 days with gentle rocking. Then it was rinsed with water and vascular cast specimens were selected and placed in a dry and ventilated place and were allowed to air-dry. The vascular cast specimens were cut into debris and metalized using the Eiko-IB-3 type ion plating machine, then they were examined under an SEM (Hitachi S-570).

\section{Statistical analysis}

The data are reported as means \pm standard deviation and were analyzed using the SPSS 12.0 software. The comparison among the groups involved the $t$-test to analyze the data. $\mathrm{P}<0.05$ was assumed to be the level of a significant difference.

\section{RESULTS}

\section{Changes in the nasal mucosal microvasculature}

SEM results on the nasal septum mucosa vascular casting in group A showed a smooth inside surface of the microartery, and capillary vessels were connected correctly with one another to form the capillary network without swollen cells or tortuous and dilated venules (Figure 1A). In group B, at 8 weeks, there were tortuous and dilated venules (Figure 1B), and there were tortuous microveins with a rough surface and aberrations at 12 weeks (Figure 1C). At 16 weeks, we found 
hyperplasia of fibrous tissue in the vessel wall and rough inner wall, swelling of vascular endothelial cells and apparent impressions in the vascular wall surface (Figure 1D).
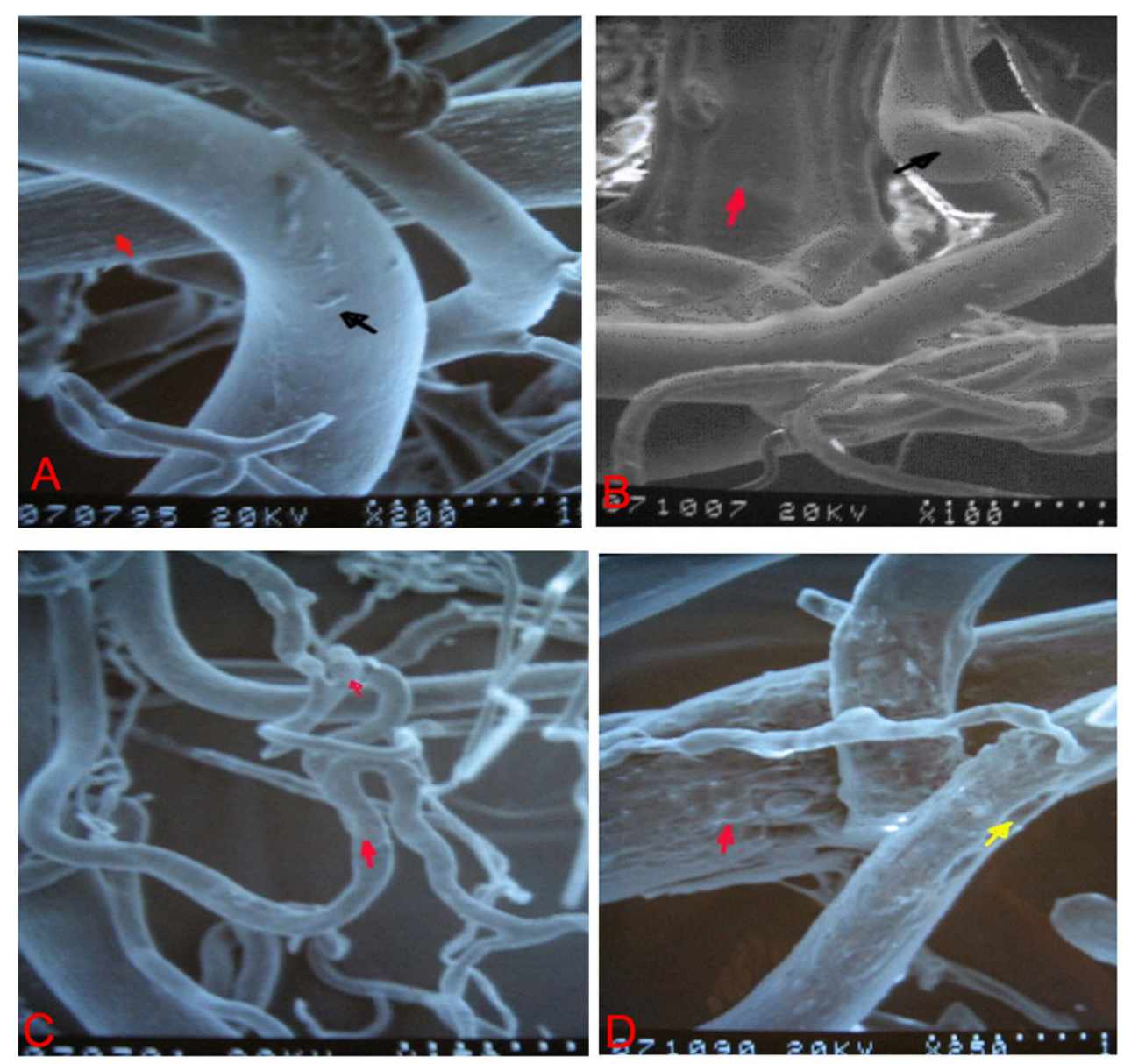

Figure 1. Scanning electron microscope results of nasal septum mucosa vascular casting in groups A and B. A. Results of group A, normal venule (black arrow), normal micro artery (red arrow). B. Results of group B at 8 weeks, the tortuous and dilated venules (black arrow), the micro artery with smooth wall (red arrow). C. Results of group B at 12 weeks, tortuous micro vein with rough surface and disorder (red arrow). D. Results of group B at 16 weeks, hyperplasia of fibrous tissue in vessel wall and rough inner wall (red arrow). Vascular endothelial cell swelling and apparent impression in vascular wall surface (yellow arrow). SEM 200X.

In group C, at 8 weeks, we observed venous congestive expansion, swelling of vascular wall cells, and aberrant proliferation of the capillary network and wall (Figure 2A); at 12 weeks, we saw the congestive expansion of microveins with a rough surface, capillary disturbances, and a pronounced vascular network (Figure 2B); at 16 weeks, venous congestive expansion, without obvious swelling of vascular wall cells as well as a dilated micro artery, a new capillary network, and a smooth wall could be seen (Figure 2C). 

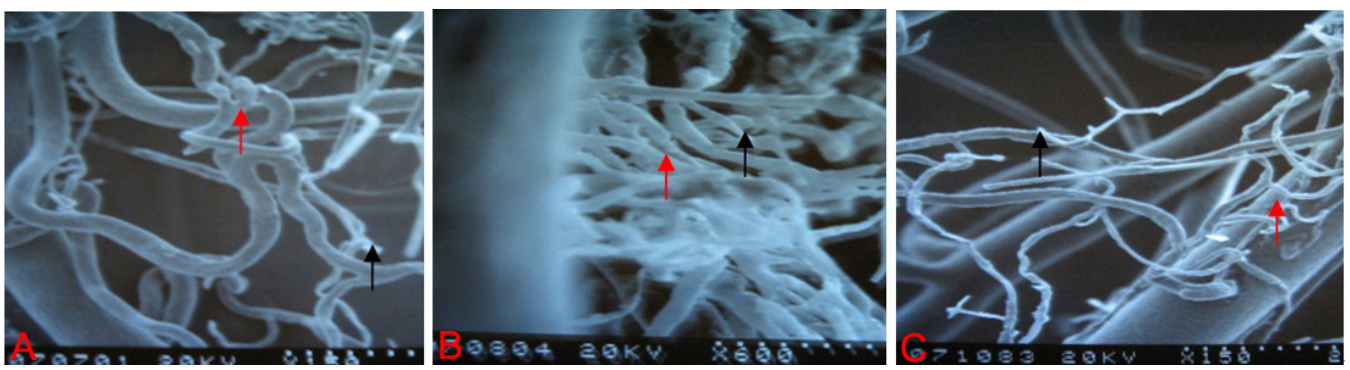

Figure 2. Scanning electron microscope results of nasal septum mucosa vascular casting in group $\mathrm{C}$. New vessels (black arrow), the capillary network and wall (red arrow). A. Results of group C at 8 weeks, venous congestive expansion, swelling vascular wall cells and disorder proliferation of capillary network and wall. B. Results of group C at 12 weeks, congestive expansion of micro vein with rough surface, capillary disorder and obvious vascular network. C. Results of group $\mathrm{C}$ at 16 weeks, venous congestive expansion, not obvious swelling vascular wall cells and dilated micro artery, new capillary network and smooth wall. SEM 200X.

\section{Expression of VEGF in the nasal septum mucosa of rats}

The expression level of VEGF in the nasal septum mucosa of group $B$ was higher than that of group $A$ while it was lower than that of group $C$ at different time points $(P<0.05)$. These data are shown in Table 1. The expression level of VEGF in the nasal septum mucosa was highest at 12 weeks in groups $B$ and $C(t=2.342, P<0.05)$.

Table 1. Comparison of VEGF expression in different groups (means \pm SD).
\begin{tabular}{lccc}
\hline Group & \multicolumn{1}{c}{ Time } & 16 week \\
\cline { 2 - 4 } & 8 week & 12 week & $0.49 \pm 0.31$ \\
\hline A & $0.57 \pm 0.10$ & $0.59 \pm 0.21$ & $1.72 \pm 0.17^{\star}$ \\
B & $1.98 \pm 0.29^{*}$ & $2.13 \pm 0.38^{*}$ & $1.31 \pm 0.10^{*}$ \\
C & $1.43 \pm 0.16^{*}$ & $1.67 \pm 0.32^{*}$ &
\end{tabular}

\section{DISCUSSION}

The ICS treatment at an early stage of AR can prevent irreversible hypothyroidism caused by airway inflammation, but cannot reverse the ongoing changes in the airway structure (Grol et al., 1999). Some researchers stated that the mechanism of ICS's action on the airway vessels includes genetic and nongenetic mechanisms (Horvath and Wanner, 2006). In the present study, we observed the effects of FP on the nasal mucosa remodeling in rats with AR.

In a previous study, we found that the nasal mucosa microvascular remodeling occurs in rats after a long-term exposure to an allergen. In this study, we found that the treatment with FP can slow down the microvascular remodeling process in the nasal mucosa; the microvascular remodeling reversed to a certain degree in the 12th week. Therefore, FP had no obvious reversal effects in the short-term, which may be one of the reasons for nasal congestion and other symptoms persisting for some time in AR after regular therapy. Thus, we hypothesized that ICS can inhibit the mucosal remodeling process of AR to a certain degree, but it had no obvious reparative effect on the preexisting irreversible change of mucosal structure. 
VEGF is known as the strongest vascular permeability factor; it can increase vascular permeability and promote the proliferation of vascular endothelial cells and vascularization. In addition, it plays a very important role in maintaining the structure and function of blood vessels and the nasal mucosal remodeling. In another study, VEGF gene was expressed in the primary bronchial and nasal polyp epithelium and the expression was affected by hypoxia (Asai et al., 2002; Jiang et al., 2002). The high expression of VEGF in epithelial cells can be self-inflicted during hypoxia and eventually lead to the formation of new blood vessels: it is also highly expressed in the airways of asthmatic patients (Boussat et al., 2000; Detoraki et al., 2010). VEGF could be highly expressed in the vascular endothelial cells of nasal polyp tissue and in inflammatory cells infiltrating the region around the vessel (Wittekindt et al., 2002). Recent studies showed that there is a causal relationship between nasal mucosa remodeling in AR and VEGF; the latter inhibits the remodeling process by the regulating the mechanism of the receptor (Moon et al., 2012). Budesonide can inhibit angiogenesis in asthma by inhibiting the expression of HIF-1a and VEGF (Sun et al., 2013). Our present results show that the expression levels of VEGF increase in AR rats, but they decreased after treatment with FP for 12 weeks. Therefore, ICS can downregulate the expression levels of VEGF in AR rats and inhibit or even reverse nasal mucosal remodeling in AR to some extent, but requires a prolonged period; this finding is consistent with the time of microvascular remodeling and morphological changes.

In conclusion, FP inhibits vascular remodeling of the nasal mucosa in AR but cannot reverse the nasal mucosal remodeling nor control VEGF expression completely in the short term.

\section{Conflicts of interest}

The authors declare no conflict of interest.

\section{ACKNOWLEDGMENTS}

We would like to thank Dr H.B. Liu, B.B Xie, and teachers of Nanchang University Electron Microscopy for their technical assistance. Research supported by the Jiangxi Provincial Science and Technology Department Support Program (\#2009BSA10200).

\section{REFERENCES}

Asai K, Kanazawa H, Otani K, Shiraishi S, et al. (2002). Imbalance between vascular endothelial growth factor and endostatin levels in induced sputum from asthmatic subjects. J. Allergy Clin. Immunol. 110: 571-575.

Barnes PJ (1995). Inhaled glucocorticoids for asthma. N. Engl. J. Med. 332: 868-875.

Boussat S, Eddahibi S, Coste A, Fataccioli V, et al. (2000). Expression of vascular endothelial growth factor in human pulmonary epithelial cells. Am. J. Physiol. Lung Cell Mol. Physiol. 279: L371-L378.

Detoraki A, Granata F, Staibano S, Rossi FW, et al. (2010). Angiogenesis and lymphangiogenesis in bronchial asthma. Allergy 65: 946-958.

Grol MH, Gerritsen J, Vonk JM, Schouten JP, et al. (1999). Risk factors for growth and decline of lung function in asthmatic individuals up to age 42 years: a 30-year follow-up study. Am. J. Respir. Crit. Care Med. 160:1830-1837.

Horvath G and Wanner A (2006). Inhaled corticosteroids: effects on the airway vasculature in bronchial asthma. Eur. Respir. J. 27: 172-187.

Jiang S, Dong Z, Zhu D and Yang Z (2002). Hypoxia effects on vascular endothelial growth factor derived epithelial cells of nasal polyps. Zhonghua Er Bi Yan Hou Ke Za Zhi. 37: 34-37.

Liu JG and Liu YH (2010). Allergic rhinitis nasal mucosa exposure to allergen histopathologic and the microvascular remodeling of SD rats. Chin. Arch. Otolaryngol. Head Neck Surg. 17: 15-17. 
Liu JG, Yang Z and Liu YH (2008). Establishment of a model of allergic rhinitis in rats. Acta Acad. Med. Jiangxi. 5: 12-13.

Lu W, Lillehoj EP and Kim KC (2005). Effects of dexamethasone on Muc5ac mucin production by primary airway goblet cells. Am. J. Physiol. Lung Cell Mol. Physiol. 288: L52-L60.

Moon IJ, Kim DY, Rhee CS, Lee CH, et al. (2012). Role of angiogenic factors in airway remodeling in an allergic rhinitis murine model. Allergy Asthma Immunol. Res. 4: 37-45.

Sun Y, Wang J, Li H, Sun L, et al. (2013). The effects of budesonide on angiogenesis in a murine asthma model. Arch. Med. Sci. 92: 361-367.

Wittekindt C, Hess A, Bloch W, Sultanie S, et al. (2002). Immunohistochemical expression of VEGF and VEGF receptors in nasal polyps as compared to normal turbinate mucosa. Eur. Arch. Otorhinolaryngol. 259: 294-298. 